\title{
The Role of Infrainguinal Bypass Surgery in the Endovascular Era
}

\author{
Raffaele Pulli, MD, Walter Dorigo, MD, Azzurra Guidotti, MD, Aaron Fargion, MD, \\ Alessandro Alessi Innocenti, MD, and Carlo Pratesi, MD
}

\begin{abstract}
The current worldwide trend in the treatment of peripheral arterial disease (PAD) is towards an increase in endovascular procedures either in the aorto-iliac area or in the infrainguinal district. However, the role of the open repair with a femoro-popliteal bypass is still debated and in our opinion there still a room for traditional surgery in the presence of complex lesions involving the superficial femoral artery and the popliteal and tibial vessels. In this field, vein bypass provides better results than prosthetic grafts, but in selected cases and not only in the absence of a suitable vein, new modified grafts may be used with satisfactory results. The choice between the two options, open and endovascular, that in some cases can be associated in hybrid procedures, depends on several factors. Only if we are able to take into account all the different preoperative issues, we could choose the right procedure in the right patient.
\end{abstract}

Keywords: peripheral artery disease, bypass surgery, endovascular procedures

The current worldwide trend in the treatment of peripheral arterial disease $(\mathrm{PAD})$ is towards an increase in endovascular procedures either in the aorto-iliac area or in the infrainguinal district. As an example, the EVEM report, one of the most popular registries in Europe for vascular and endovascular interventions, recently reported a significant increase of endovascular approach for infrainguinal disease in the recent years.

The increasing trend in favor of the endovascular techniques has probably to be attributed to two main factors. In first instance, young vascular surgeons are more prone towards the new technologies and their training is easier and shorter than that requested for performing traditional interventions; the second

\section{Department of Vascular Surgery, University of Florence, Florence, Italy}

Received: December 16, 2013; Accepted: December 19, 2013 Corresponding author: Raffaele Pulli, MD. Department of Vascular Surgery, University of Florence, Largo Brambilla 3, Florence 50134, Italy

Tel: +39-055-7947607, FAX: +39-055-7947448

E-mail:rpulli@unifi.it issue is the request and the better acceptance by the patients of a less invasive endovascular procedure.

Clearly, the two options, vascular and endovascular, should not be completely alternative and the surgeons should be able to choose the best procedure in the single patient. In addition, the possibility of performing hybrid interventions, which means to use the two techniques in the same patient during the same operation, represents another opportunity for the surgeon.

The hybrid intervention is used when an endovascular approach alone is not feasible or it is supposed to be unable to warrant a satisfactory result. Typical examples are the endarterectomy of common femoral artery associated with stenting of the iliac axis or with angioplasty of the superficial femoral artery and the femoropopliteal bypass associated with the angioplasty of the tibial vessels, in the latter case in order to improve the outflow.

Another important issue of the endovascular techniques is their possible role in maintaining the patency of the so called failing bypass grafts. The possibility to treat with an endovascular procedure an anastomotic stenosis before it leads to graft occlusion allows to prevent a redo surgery that would be more complex and technically demanding. 
In the attempt to define the indications and the limits on the treatment of peripheral artery disease, the TASC classification has been created and updated. It divides the aorto-iliac localizations from the infrainguinal ones and each area is divided into classes $\mathrm{A}, \mathrm{B}$, $\mathrm{C}$ and $\mathrm{D}$. In class $\mathrm{A}$ the endovascular treatment is strongly recommended, while in class D surgical treatment is strongly recommended. In classes B and C a primary approach with endovascular or open surgical repair is suggested, respectively. ${ }^{1)}$

As far as the infrainguinal diseases are concerned, the class A includes short stenoses or occlusions of the superficial femoral artery, while the class B includes short multiple or longer single lesions. The class $\mathrm{C}$ comprises multiple or single long lesions of the superficial femoral artery, while class D comprises long occlusions of SFA involving also popliteal or tibial vessels.

Several studies have shown that patients with critical limb ischemia have an increased risk of initial failure compared with claudicants, probably due to severity of the disease and the consequent complexity of the surgical techniques. ${ }^{2)}$ Endovascular surgery in these patients may represent a first line treatment, due to the less invasiveness of the procedure, leaving open surgery as an alternative option for the future. In this sense it is of paramount importance the behavior of the physician who performs the endovascular procedure. In other words, it is crucial to preserve a healthy distal arterial segment which can be used as the distal anastomotic site for a future surgical bypass in the case of an endovascular failure. ${ }^{3)}$

Regarding some specific issues, it is still rather controversial the use of stents in SFA, clinical studies having not yet shown their effectiveness in the treatment of superficial femoral artery occlusions. ${ }^{4)}$ Furthermore, the endovascular treatment in young patients $^{5)}$ and in women ${ }^{6)}$ is questionable, being a statistically significant difference in term of primary and secondary patency in comparison with elderly and men.

Even in the presence of such a large diffusion of endovascular techniques in patients with PAD, a significant number of patients still requires a surgical bypass due the complexity of arterial lesions or following the failure of a primary endovascular attempt. Open surgery has been demonstrated to provide good and long term results, even if the rate of late complications in terms of graft failure is still not negligible.

Among the factors affecting the patency of femoropopliteal bypasses, the clinical presentation, the level of distal anastomosis, the graft material and the status of the outflow vessels seem to be preponderant. Several studies have shown that the femoropopliteal prosthetic grafts have a 5-year patency rate significantly lower than the vein bypasses, and it is commonly accepted that the vein performs better than prosthetic grafts and should be used whenever possible. ${ }^{7)}$

When a vein bypass fails, the causes of such a failure can depend on patient's characteristics (demographic, comorbidity, genetics), on altered biological response (proliferation of smooth muscle cells, vascular remodeling, inflammation, functionality of endothelium) and on technical factors (quality and caliber of the vein, inflow and outflow status, technical errors). $\left.{ }^{8}\right)$

Recently, a basic science study has shown that a mutation of the p27 gene can be associated with the outcome of the surgical bypasses.9) Other authors tried to create a prognostic score for the saphenous vein bypass (CRAB); the considered factors were: age $>75$ years, prior amputation/revascularization, ulceration, hemodialysis, recent angina, emergent case, partial/total functional dependence. ${ }^{10)}$

On the other hand, the main factor associated with late failure of prosthetic graft has been supposed to be dependent on the intrinsic thrombogenicity of synthetic materials, along with the miointimal hyperplasia at the anastomotic sites and the progression of atherosclerosis on inflow and outflow vessels.

In order to improve the patency rate of the prosthetic grafts, some modifications, either morphological, such as a precuffed graft for the distal anastomosis, or biochemical, in particular with the introduction of an heparin-bonded surface, have been proposed. ${ }^{11}$

In Italy, a multicentric graft-based registry on the use of heparin-bonded ePTFE grafts has been working since 2002. Seven centers are involved, with the aim to assess the early and long-term results of this graft. Being a registry, neither exclusion nor inclusion criteria were required, and the choice of the graft was at the surgeon's discretion and not only in the absence of a suitable saphenous vein.

The results of such registry, which have been published in several international journals, ${ }^{12-14)}$ are satisfactory in the early and long term settings, showing 
primary and secondary patency rates at 7 years of more than $40 \%$ and $50 \%$, respectively. Even if such values are still poorer than those obtained with autologous vein in the same centers in the same period of time, no differences were found with vein bypasses in terms of amputation-free survival.

A subgroup analysis is on the way in order to create a score for predicting the results of heparinbonded grafts and for possibly detecting those patients who can primarily benefit from a prosthetic graft even in the presence of a suitable autologous vein. Preliminary data seem to indicate that in highly selected male patients undergoing primary below-knee intervention, with rest pain rather than ulcers and at least two patent tibial vessels, the results of heparin bonded graft are similar to those of autologus vein in terms of primary and secondary patency and amputation-free survival.

Regardless the choice of the appropriate technique and of the material for revascularization, a careful analysis of the clinical status, of the morphology of the lesions and of local and systemic surgical risk, along with the experience and the surgical volume of the operators and of the hospital are at the moment the main issues to be considered when planning an invasive procedure in the infrainguinal district. ${ }^{15)}$

Probably in the future another crucial point will be the impact of the procedure on the quality of life of the patient. Preliminary findings ${ }^{16)}$ seem to demonstrate that open surgery for PAD provides a significant improvement in health status and quality of life in the long-term follow-up. On the other hand, endovascular treatment seems to give better early satisfaction because the patient does not suffer from immediate social restrictions and has a general perception of a better physical status in comparison with open surgery.

\section{Conclusions}

In the era of endovascular procedures, bypass surgery still represents a safe and feasible option in patients with complex femoro-popliteal lesions requiring technically demanding procedures. In patients requiring a surgical bypass for critical limb ischemia, the autologous vein remains the material of choice. The role of modified prosthetic grafts is still under investigation: they can represent an effective alternative to autologous vein when it is absent or of poor quality and probably their use can be suggested as initial treatment in highly selected patients.

\section{Disclosure Statement}

There is no conflict of interest for this article.

\section{References}

1) Norgren L, Hiatt WR, Dormandy JA, et al. Inter-Society Consensus for the Management of Peripheral Arterial Disease (TASC II). J Vasc Surg 2007; 45 Suppl S: S5-67.

2) DeRubertis BG, Pierce M, Chaer RA, et al. Lesion severity and treatment complexity are associated with outcome after percutaneous infra-inguinal intervention. J Vasc Surg 2007; 46: 709-16.

3) Joels CS, York JW, Kalbaugh CA, et al. Surgical implications of early failed endovascular intervention of the superficial femoral artery. J Vasc Surg 2008; 47: 562-5.

4) Soga Y, Iida O, Hirano K, et al. Mid-term clinical outcome and predictors of vessel patency after femoropopliteal stenting with self-expandable nitinol stent. J Vasc Surg 2010; 52: 608-15.

5) Chaar CI, Makaroun MS, Marone LK, et al. Impact of endovascular options on lower extremity revascularization in young patients. J Vasc Surg 2012; 56: 703-13. e1-3.

6) Pulli R, Dorigo W, Pratesi G, et al. Gender-related outcomes in the endovascular treatment of infrainguinal arterial obstructive disease. J Vasc Surg 2012; 55: 105-12.

7) Pereira CE, Albers M, Romiti M, et al. Meta-analysis of femoropopliteal bypass grafts for lower extremity arterial insufficiency. J Vasc Surg 2006; 44: 510-7.

8) Monahan TS, Owens CD. Risk factors for lower-extremity vein graft failure. Semin Vasc Surg 2009; 22: 216-26.

9) Conte MS, Owens CD, Belkin M, et al. A single nucleotide polymorphism in the p27(Kip1) gene is associated with primary patency of lower extremity vein bypass grafts. J Vasc Surg 2013; 57: 1179-85.e1-2.

10) Meltzer AJ, Graham A, Connolly PH, et al. The Comprehensive Risk Assessment for Bypass (CRAB) facilitates efficient perioperative risk assessment for patients with critical limb ischemia. J Vasc Surg 2013; 57: 1186-95.

11) Dorigo W, Di Carlo F, Troisi N, et al. Lower limb revascularization with a new bioactive prosthetic graft: early and late results. Ann Vasc Surg 2008; 22: 79-87.

12) Pulli R, Dorigo W, Castelli $P$, et al. Midterm results from a multicenter registry on the treatment of infrainguinal critical limb ischemia using a heparin-bonded ePTFE graft. J Vasc Surg 2010; 51: 1167-77.e1.

13) Dorigo W, Pulli R, Castelli P, et al. A multicenter comparison between autologous saphenous vein and heparin-bonded expanded polytetrafluoroethylene (ePTFE) 
graft in the treatment of critical limb ischemia in diabetics. J Vasc Surg 2011; 54: 1332-8.

14) Dorigo W, Pulli R, Piffaretti G, et al. Results from an Italian multicentric registry comparing heparin-bonded ePTFE graft and autologous saphenous vein in belowknee femoro-popliteal bypasses. J Cardiovasc Surg (Torino) 2012; 53: 187-94.
15) Conte MS. Critical appraisal of surgical revascularization for critical limb ischemia. J Vasc Surg 2013; 57: 8S-13S.

16) Pratesi C, Taddei S, Compiani E, et al. Short-term patient-reported outcomes of open and endovascular treatment of peripheral arterial disease. J Vasc Surg 2010: 51: 61S. 